\title{
Ultrasonic features of papillary thyroid microcarcinoma coexisting with a thyroid abnormality
}

\author{
BO LI ${ }^{1}$, YAQIONG ZHANG ${ }^{2}$, PING YIN ${ }^{1}$, JIAN ZHOU ${ }^{1}$ and TIAN'AN JIANG ${ }^{3}$ \\ ${ }^{1}$ Department of Ultrasound, Taizhou Municipal Hospital, Medical College of Taizhou University, Taizhou, Zhejiang 318000; \\ ${ }^{2}$ Department of Clinical Laboratory, Taizhou Central Hospital, Taizhou, Zhejiang 318000; ${ }^{3}$ Department of Ultrasound, \\ The First Affiliated Hospital of Zhejiang University College of Medicine, Hangzhou, Zhejiang 310003, P.R. China
}

Received April 11, 2015; Accepted July 12, 2016

DOI: $10.3892 /$ ol.2016.4999

\begin{abstract}
The present study aimed to investigate the value of ultrasonography in the diagnosis of papillary thyroid microcarcinoma (PTMC) coexisting with a thyroid abnormality, and to improve the accuracy of PTMC diagnosis. The ultrasonic features of 38 PTMC nodules coexisting with a thyroid abnormality and 56 thyroid benign nodules, obtained by surgical resection and confirmed by pathological analysis, were retrospectively analyzed. All masses were $\leq 1.0 \mathrm{~cm}$ in diameter. Ultrasonic features that were analyzed included the shape, aspect ratio, boundary, margin, echo, uniformity, presence or absence of microcalcification and enlargement of the lymph nodes, as well as the blood flow of the nodules. Furthermore, the sensitivity, specificity and accuracy of ultrasonography for the diagnosis of PTMC were obtained. The following ultrasonic features of thyroid nodules were significantly $(\mathrm{P}<0.05)$ associated with PTMC coexisting with a thyroid abnormality: An irregular shape; an aspect ratio of $\geq 1$; an unclear boundary; blurred margins; internal heterogeneous hypoechogenicity; and microcalcification. Therefore, thyroid nodules with these ultrasonic characteristics coexisting with a thyroid abnormality may be suspected as malignant PTMC. The present study demonstrated that ultrasound-guided biopsies are necessary to prevent misdiagnosis of PTMC. The sensitivities of enlarged neck lymph nodes and abundant blood flow are so low that they may be considered as references for the differentiation of PTMC from benign nodules.
\end{abstract}

Correspondence to: Professor Tian'an Jiang, Department of Ultrasound, The First Affiliated Hospital of Zhejiang University College of Medicine, 79 Qingchun Road, Hangzhou, Zhejiang 310003, P.R. China

E-mail: tangtan0531@yeah.net

Key words: papillary thyroid microcarcinoma, thyroid abnormality, ultrasonic diagnosis

\section{Introduction}

Papillary thyroid microcarcinoma (PTMC), which is a particular variant of papillary thyroid carcinoma, is defined as a papillary thyroid cancer measuring $<1.0 \mathrm{~cm}$ at its maximal diameter and accounts for $\sim 85 \%$ of thyroid tumors in the United States (1). To date, the diagnosis of PTMC has been highly reliant on high frequency ultrasound examination. Clinical outcomes for patients with PTMC are excellent; the 10 -year survival rate for patients with PTMC is $>90 \%$ (2). However, the preoperative diagnostic rate is low, and PTMC is often misdiagnosed due to its slow growth rate, absence of specific symptoms or clinical characteristics and potential co-occurrence with benign thyroid diseases (3). Currently, the most effective treatment for PTMC remains disputed. Certain researchers propose a "wait and see" approach until the tumor reaches a large size, while others propose early treatment following diagnosis $(4,5)$. Although there have been a number of reports regarding the ultrasonic diagnosis of PTMC in China and other countries $(6,7)$, few cases of PTMC associated with a thyroid abnormality (including primary hyperthyroidism, Hashimoto's thyroiditis and nodular goiter) have been diagnosed using ultrasonography (8-10).

In the present study, 38 nodules that were isolated from the thyroid glands of patients with PTMC and coexisting benign thyroid diseases between April 2010 and September 2013 were retrospectively analyzed. Of these nodules, 21 coexisted with Hashimoto's thyroiditis (HT), 10 with nodular goiter and 7 with hyperthyroidism (PTH). Furthermore, ultrasonic images of 56 nodules from patients with benign nodular goiter were used as controls. The present study aimed to investigate the value of ultrasonography in the diagnosis of PTMC coexisting with a thyroid abnormality, and to improve the accuracy of PTMC diagnosis.

\section{Materials and methods}

Patients and nodule collection. The present study was approved by the Ethics Committee of the Municipal Hospital of Taizhou University School of Medicine (Taizhou, China), and written informed consent was obtained from all patients. The present study retrospectively analyzed 94 nodules collected from patients with thyroid disorders at the Municipal 
Hospital of Taizhou University School of Medicine between April 2010 and September 2013. The thyroid disorders had been diagnosed with high frequency ultrasonography, and confirmed by surgical resection and pathological examination. Of these patients, 34 were men and 60 were women, and 38 were diagnosed with PTMC and 56 with benign nodular goiter, which were considered as the controls. The mean age of the patients was 45 years (range, 15-86 years). Patients with PTMC or benign thyroid disease who could not tolerate, or were not willing to receive, surgery were excluded from the present study.

Instruments and imaging procedures. All ultrasound examinations were performed with the Acuson Sequoia-512 (Siemens AG, Munich, Germany) or the Toshiba SSA-770A Color Doppler instrument (Toshiba Corporation, Tokyo, Japan) equipped with a 5-12 MHz linear probe. For ultrasound of the thyroid nodules, the lobes and isthmus of the thyroid gland were sectioned and examined to observe the shape, boundary, margin, echotexture, microcalcification and intranodular or surrounding vascularity.

According to the latest guidelines for Ultrasound on Thyroid Gland in Chinese Physicians Branch of Ultrasound Doctor (11), the following eight aspects of the nodules were analyzed: i) Shape, in particular whether a nodule was regular or irregular (a nodule was deemed regular when it was ovoid or round); ii) aspect ratio, in particular, whether the aspect ratio was more than or less than one (the aspect ratio was defined as the ratio of the anteroposterior diameter to the transverse diameter of the nodule); iii) boundary, in particular, whether the boundary of a nodule was clear or unclear (a nodule had a clear boundary when there was a definite boundary between the nodule and peripheral tissue); iv) margin, in particular whether a margin was smooth or rough (smoothness was defined when there was a clear change between the nodule and peripheral tissue, whereas the margin was rough when the nodule was angulated, lobulate, blurred or obscured); v) echogenicity, specifically, whether a nodule showed intranodular hypoechogenicity or non-hypoechogenicity (the echogenicity of a nodule with respect to the adjacent tissue was classified as hypoechoic, isoechoic, hyperechoic or mixed echoic); vi) uniformity of the echotexture, in particular whether it was homogeneous or heterogeneous (a heterogeneous nodule was defined as having varying echoic levels); vii) the presence or absence of microcalcification (microcalcification was deemed present when there were several strong, punctate echogenic foci of $\leq 2 \mathrm{~mm}$ that were distributed as clusters or scattering spots); and viii) vascularity type, according to the distribution of blood flow in the nodule (vascularity patterns were determined to be mixed type, no vascular type, peripheral type or central type).

Statistical analysis. Statistical analyses were performed using SPSS 18.0 software (SPSS, Inc., Chicago, IL, USA). Student's t-tests were used for comparing quantitative variables between PTMC and benign control groups, and $\chi^{2}$-tests were used to compare two-dimensional images. $\mathrm{P}<0.05$ was considered to indicate a statistically significant difference.

\section{Results}

Histopathological examination. The diagnosis of PTMC via histological examination included 29 isolated nodules and

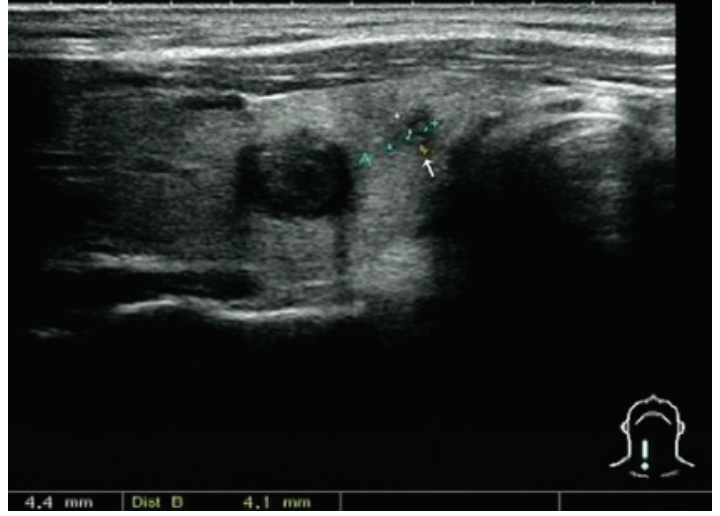

Figure 1. An ultrasound scan of the right thyroid lobe of a patient with papillary thyroid microcarcinoma and coexisting thyroid disease shows a hypoechoic nodule.

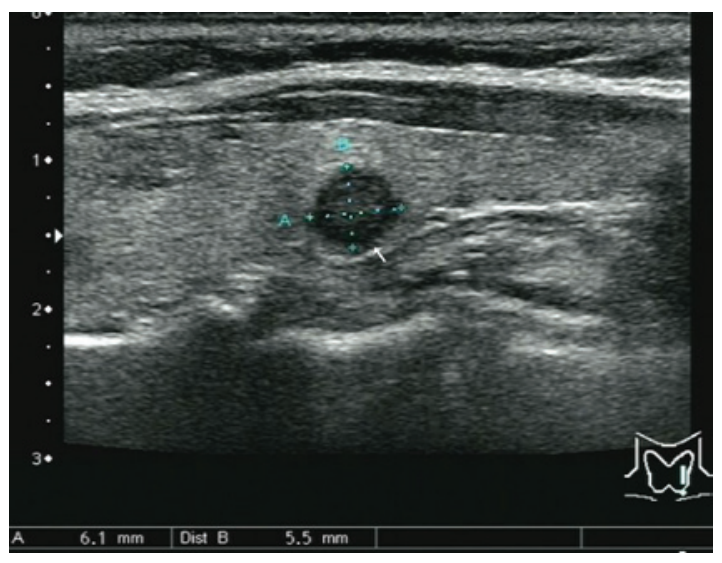

Figure 2. An ultrasound scan of the left thyroid lobe of a patient with papillary thyroid microcarcinoma and a coexisting thyroid disease shows a hyperechoic nodule.

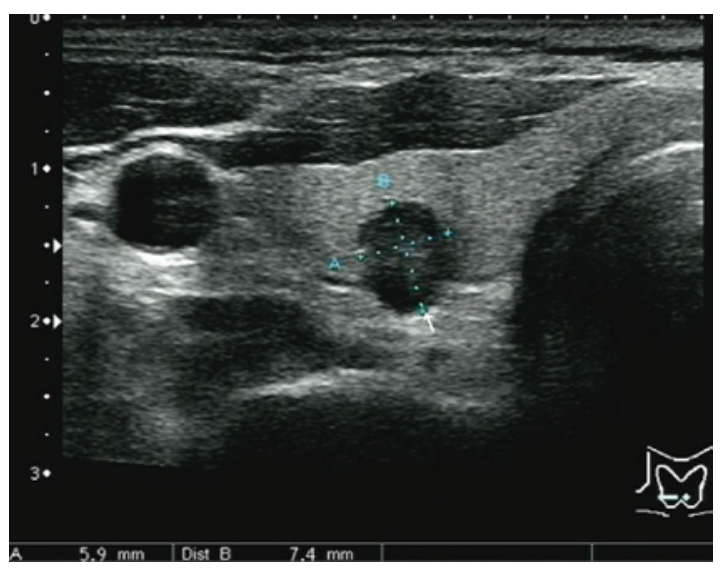

Figure 3. An ultrasound scan of the right thyroid lobe of a patient with papillary thyroid microcarcinoma and coexisting hyperthyroidism shows a nodule with microcalcification.

9 double focal nodules ( 3 unilateral and 6 bilateral multifocal nodules; in cases with multiple lesions, the maximal lesion was investigated in the present study). A total of 38 nodules derived from the thyroid glands of patients with PTMC were associated with benign thyroid diseases. Of these nodules, 21 were associated with HT, 10 with nodular goiter and 7 with PTH. 


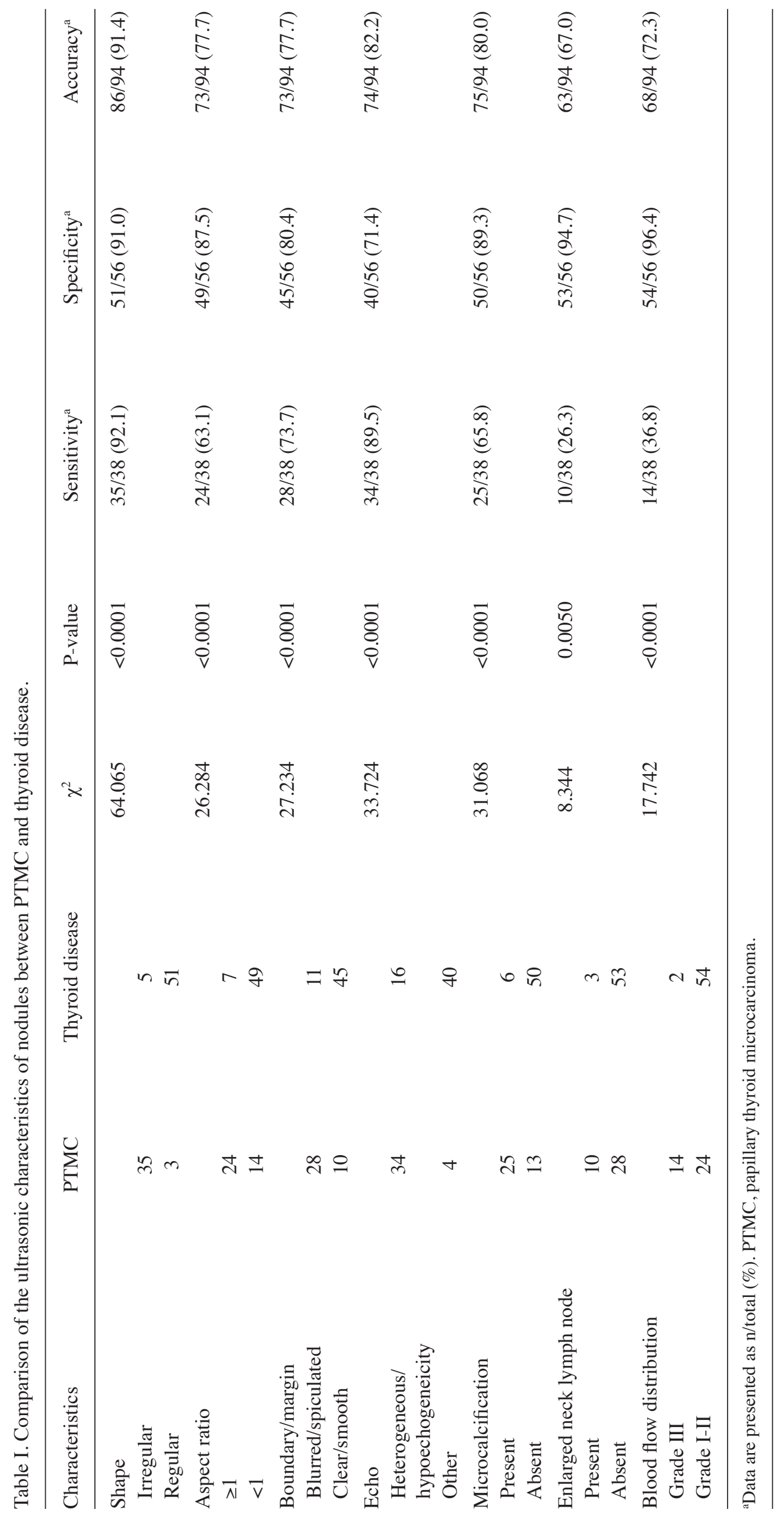




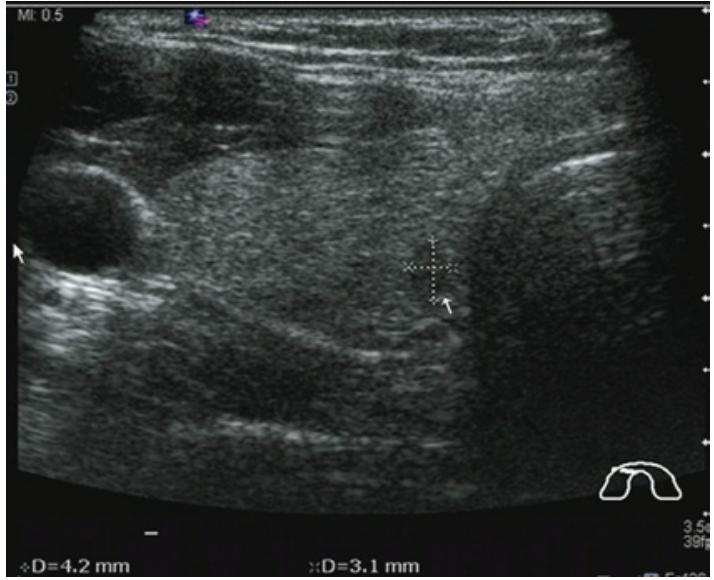

Figure 4. An ultrasound scan of the right thyroid lobe of a patient with papillary thyroid microcarcinoma and coexisting hyperthyroidism.

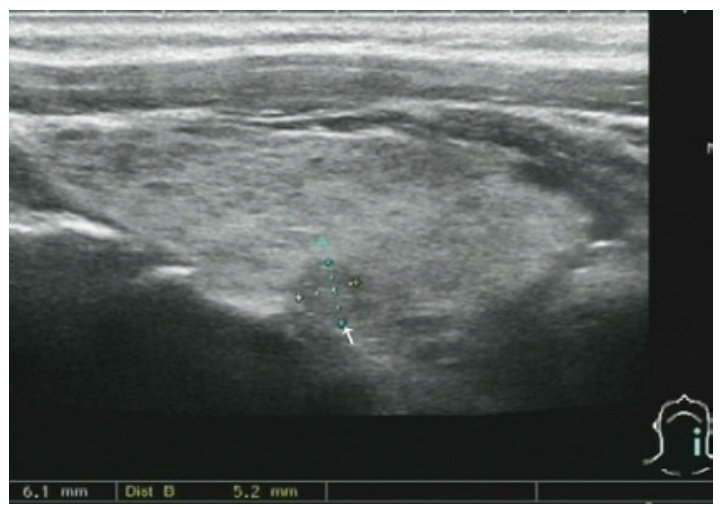

Figure 5. An ultrasound scan of the right thyroid lobe of a patient with papillary thyroid microcarcinoma and coexisting Hashimoto thyroiditis shows a nodule with microcalcification.

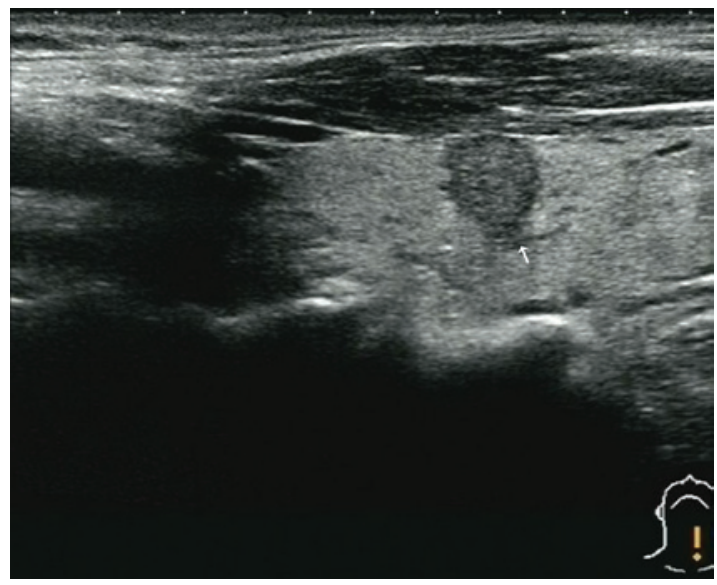

Figure 6. An ultrasound scan of the left thyroid lobe of a patient with papillary thyroid microcarcinoma and coexisting Hashimoto thyroiditis.

Ultrasonic characteristics of all nodules. A comparison of the ultrasonic results for PTMC and benign nodules is shown in Table I. The features that were significantly associated with PTMC included an irregular shape, an aspect ratio of $>1$, a blurred boundary, a spiculated margin, heterogeneous hypoechogenicity, the presence of microcalcification and

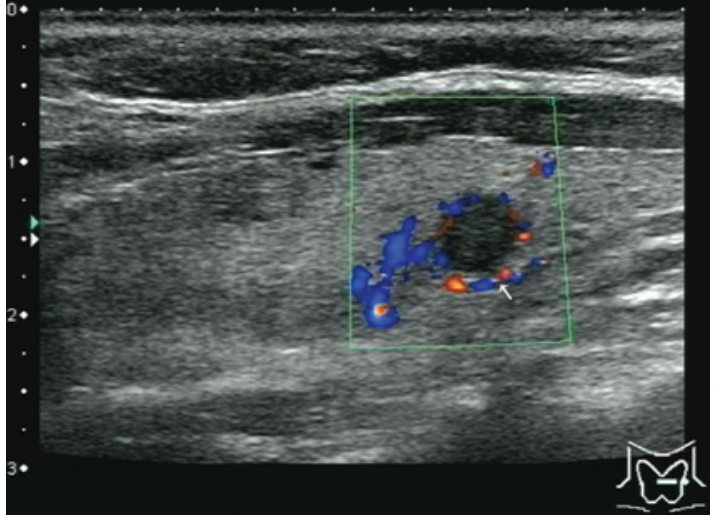

Figure 7. An ultrasound scan of the left thyroid lobe of a patient with papillary thyroid microcarcinoma shows a nodule with a secondary blood supply.

enlarged neck lymph nodes, as well as the abundant distribution of blood flow. Furthermore, these features showed a relatively high specificity and diagnostic accuracy $(\mathrm{P}<0.05)$, although the presence of enlarged neck lymph nodes and the abundant distribution of blood flow did not show a high sensitivity. Figs. 1-7 reveal further information about the nodules.

\section{Discussion}

Ultrasonography, as the preferred imaging technique, has been widely used to differentiate PTMC from benign nodules; however, the majority of patients with PTMC have malignant nodules (3). In the present study, the ultrasonic features of malignant PTMC, compared with benign nodules, were an irregular shape, an aspect ratio of $>1$, an unclear boundary, a spiculated margin, heterogeneous hypoechogenicity, microcalcification, the enlargement of neck lymph nodes and the abundant distribution of blood flow. These characteristics could easily differentiate PTMC from non-tumor lesions and benign nodules. However, various types of PTMC have been shown to coexist with other thyroid disease, rather than independently. Therefore, the background ultrasonic images of the thyroid parenchyma may effect the diagnosis of tumors. The echogenicity of the normal thyroid parenchyma is intensive and homogeneous compared with the isoechoic pattern of anterior muscle and thyroid contours (10). Once the echogenicity of the thyroid parenchyma becomes thickened, heterogeneous and shows a nodular focus, diagnosing the disease using ultrasonic images becomes a challenge (12). Due to a lack of understanding of PTMC, primarily due to non-specific symptoms that may also indicate benign thyroid disease, the accuracy rate of preoperative diagnosis is very low. Therefore, investigating the ultrasonic features of PTMC and the causes of misdiagnosis will improve the accuracy rate of preoperative diagnosis.

HT and PTH are characterized by a reduced, thickened and heterogeneous echogenicity of the thyroid parenchyma, which can be classified into diffuse, nodular and restrictive types (13). Among these types, ultrasonic images of the nodular pattern are complex and are easily mistaken for thyroid malignant disease. Cases of nodular HT, PTH and PTMC coexisting with HT or PTH nodules require differentiation from benign nodules with HT or PTH. HT or PTH coexisting with a thyroid 
adenoma is characterized by an intact envelope, a regular shape, peripheral hypoechogenicity, an internal isoechoic or hyperechoic pattern and the absence of microcalcification. When HT or PTH coexist with nodular goiter, the majority of nodules are heterogeneous and either cystic-solid, solid or cystic, and macrocalcification is present. The nodules of patients with PTMC coexisting with HT or PTH have an irregular shape, blurred margin, internal hypoechogenicity, an aspect ratio of $>1$ and microcalcification. In the present study, 7 nodules were characterized by a clear boundary and an isoechoic pattern, which could be confused with a benign nodule. However, the ultrasonic features included intranodular microcalcification and an abundant blood flow, which could be used to differentiate from a benign nodule. A previous study demonstrated that HT with coexisting thyroid cancer showed much coarser calcification, but less microcalcification compared with HT without co-occurrence of a malignant cancer (14). The results of the present suggested that the incidence of coarse calcification is uncommon ( 2 cases of HT nodules coexisting with PTMC) compared with microcalcification (13 cases of HT nodules coexisting with PTMC). These results may have been due to the small sample size and bias associated with the selection of surgical patients.

PTMC is often clinically undetectable in cases of PTMC coexisting with nodular goiter due to its small size and deep localization in the thyroid gland; however, nodules with a diameter of 2-3 $\mathrm{mm}$ can be detected with the use of high-resolution transducers (3). In addition, certain features of nodules make the diagnosis of PTMC a challenge, including a lack of specific ultrasonic characteristics when combined with nodular goiter, multifocal pattern (13.33\% tumors of multifocal nodules) and complex and irregular ultrasonic images. Therefore, radiologists should assess every nodule in patients with nodular goiter, in particular hypoechoic solid nodules, by multi-section imaging. Furthermore, the following features of a nodules may aid in the diagnosis of PTMC in patients with a background of nodular goiter: i) The absence of a clear boundary without an evident halo; ii) an aspect ratio of $\geq 1$; iii) the presence of microcalcification; iv) an abundant blood flow; and v) an unexplained enlargement of the lymph node, in particular in the nodes of the middle and lower neck, and paratracheal lymph node (15).

In a previous study, the detection rate of PTMC was significantly decreased when it coexisted with nodular goiter, HT or PTH compared with PTMC alone, since atypical nodules may be ignored when scanning multiple foci (16). Potentially the most important features for determining a diagnosis of PTMC include: i) The size of the PTMC nodule is small and the lesion appears during the early stages of the disease. In addition, infiltration of tumor cells into the adjacent gland is not evident, and certain PTMC nodules have a regular shape $(8.0 \%$ in the present study). ii) The internal blood flow of PTMC is significantly reduced compared with thyroid cancer with a diameter of $>1.0 \mathrm{~mm}$ (15). In the present study, the blood flow of PTMC nodules was predominantly grade I-II according to the Adler grading system, accounting for $24(63.2 \%)$ of the nodules, whereas only $14(36.8 \%)$ of the nodules were grade III (17). This difference may be due to the fact that the growth of PTMC nodules is slow, novel blood vessels are few and the vascular structure resembles normal vessels (18). However, given the high specificity, the blood distribution in PTMC nodules may be considered an important indicator for the diagnosis of PTMC. PTMC often coexists with benign nodules and the pathogenesis of PTMC is not well-elucidated; thus, benign nodules are more readily diagnosed compared with PTMC nodules, leading to the misdiagnosis of PTMC.

In conclusion, the present study demonstrated that the ultrasonic characteristics of PTMC nodules include an irregular shape, an aspect ratio of $>1$, a blurred boundary, a spiculated margin, heterogeneous hypoechogenicity, the presence of microcalcification, enlargement of neck lymph nodes and an abundant distribution of blood flow. High-resolution ultrasound has an important value in the diagnosis and preoperative assessment of PTMC, and is able to provide high-quality images compared with computed tomography or magnetic resonance imaging (3). The results of the present study suggest that the presence of small nodules with one or more of the aforementioned characteristics should be suspected as a malignant nodule and PTMC. According to the guidelines proposed by the American Thyroid Association, to prevent misdiagnosis an ultrasound-guided fine-needle aspiration biopsy may be performed (19). The sensitivities of enlarged neck lymph nodes and the abundant distribution of blood flow are so low that they may be considered as references to permit the differentiation of PTMC from benign nodules.

\section{References}

1. Sugitani I, Kasai N, Fujimoto Y and Yanagisawa A: A novel classification system for patients with PTC: Addition of the new variables of large $(3 \mathrm{~cm}$ or greater) nodal metastases and reclassification during the follow-up period. Surgery 135: 139-148, 2004.

2. McHenry CR and Phitayakorn R: Follicular adenoma and carcinoma of the thyroid gland. Oncologist 16: 585-593, 2011.

3. Chen HY, Liu WY, Zhu H, Jiang DW, Wang DH, Chen Y, Li W and Pan G: Diagnostic value of contrast-enhanced ultrasound in papillary thyroid microcarcinoma. Exp Ther Med 11: 1555-1562, 2016.

4. Ito $\mathrm{Y}$, Miyauchi A, Inoue $\mathrm{H}$, Fukushima $\mathrm{M}$, Kihara $\mathrm{M}$, Higashiyama T, Tomoda C, Takamura Y, Kobayashi K and Miya A: An observational trial for papillary thyroid microcarcinoma in Japanese patients. World J Surg 34: 28-35, 2010.

5. Kim HY, Park WY, Lee KE, Park WS, Chung YS, Cho SJ and Youn YK: Comparative analysis of gene expression profiles of papillary thyroid microcarcinoma and papillary thyroid carcinoma. J Cancer Res Ther 6: 452-457, 2010.

6. Li QS, Chen SH, Xiong HH, Xu XH, Li ZZ and Guo GQ: Papillary thyroid carcinoma on sonography. Clin Imaging 34: 121-126, 2010

7. Moon WJ, Jung SL, Lee JH, Na DG, Baek JH, Lee YH, Kim J, Kim HS, Byun JS and Lee DH; Thyroid Study Group, Korean Society of Neuro- and Head and Neck Radiology: Benign and malignant thyroid nodules: US differentiation-multicenter retrospective study. Radiology 247: 762-770, 2008.

8. Wu X, Yu J, Kang WM, Ma ZQ and Ye X: Surgical diagnosis and treatment of primary hyperthyroidism complicated with occult thyroid carcinoma. Zhongguo Yi Xue Ke Xue Yuan Xue Bao 37: 402-405, 2015

9. Konturek A, Barczyński M, Wierzchowski W, Stopa M and Nowak W: Coexistence of papillary thyroid cancer with Hashimoto thyroiditis. Langenbecks Arch Surg 398: 389-394, 2013.

10. Gul K, Dirikoc A, Kiyak G, Ersoy PE, Ugras NS, Ersoy R and Cakir B: The association between thyroid carcinoma and Hashimoto's thyroiditis: The ultrasonographic and histopathologic characteristics of malignant nodules. Thyroid 20 : 873-878, 2010.

11. Li F, Zhang J, Wang Y and Liu L: Clinical value of elasticity imaging and contrast-enhanced ultrasound in the diagnosis of papillary thyroid microcarcinoma. Oncol Lett 10: 1371-1377, 2015. 
12. Chaudhary V and Bano S: Thyroid ultrasound. Indian J Endocrinol Metab 17: 219-227, 2013.

13. Khati N, Adamson T, Johnson KS and Hill MC: Ultrasound of the thyroid and parathyroid glands. Ultrasound Q 19: 162-176, 2003.

14. Ohmori N, Miyakawa M, Ohmori $\mathrm{K}$ and Takano $\mathrm{K}$ : Ultrasonographic findings of papillary thyroid carcinoma with Hashimoto's thyroiditis. Intern Med 46: 547-550, 2006.

15. Anil G, Hegde A and Chong FH: Thyroid nodules: Risk stratification for malignancy with ultrasound and guided biopsy. Cancer Imaging 11: 209-223, 2011

16. Ito Y, Miyauchi A, Kihara M, Higashiyama T, Kobayashi K and Miya A: Patient age is significantly related to the progression of papillary microcarcinoma of the thyroid under observation. Thyroid 24: 27-34, 2014.
17. Adler DD, Carson PL, Rubin JM and Quinn-Reid D: Doppler ultrasound color flow imaging in the study of breast cancer: Preliminary findings. Ultrasound Med Biol 16: 553-559, 1990.

18. Pettersson A, Nagy JA, Brown LF, Sundberg C, Morgan E, Jungles S, Carter R, Krieger JE, Manseau EJ, Harvey VS, et al: Heterogeneity of the angiogenic response induced in different normal adult tissues by vascular permeability factor/vascular endothelial growth factor. Lab Invest 80: 99-115, 2000.

19. American Thyroid Association (ATA) Guidelines Taskforce on Thyroid Nodules and Differentiated Thyroid Cancer, Cooper DS, Doherty GM, Haugen BR, Kloos RT, Lee SL, Mandel SJ, Mazzaferri EL, McIver B, Pacini F, et al: Revised American Thyroid Association management guidelines for patients with thyroid nodules and differentiated thyroid cancer. Thyroid 19: $1167-1214,2009$. 